\title{
A Systematic Review and Meta-analysis
}

${ }^{1}$ Tristan Struja, ${ }^{1}$ Larissa Guebelin, ${ }^{1}$ Alexander Kutz, ${ }^{1}$ Hannah Fehlberg, ${ }^{1,}$ 'Beat Mueller, ${ }^{1,}$ 2Philipp Schuetz

${ }^{1}$ Medical University Department, Clinic for Endocrinology, Diabetes \& Metabolism, Kantonsspital Aarau, Aarau, Switzerland

2 Medical Faculty of the University of Basel, Basel, Switzerland

\section{Introduction}

Graves' disease is an autoimmune disorder and common cause of primary hyperthyroidism. The overall prevalence of Graves' disease varies but is $0.5 \%$ (1). A standard treatment for Graves' disease includes use of thyrostatic drugs for a total duration of 12 to 18 months to treat overproduction of thyroid hormones. Yet, this treatment does not have an effect on the underlying autoimmune disorder, and risk of relapse is around $50 \%$ (2). Because of the autoimmune nature of Graves' disease, additional use of immunosuppressive drugs, in combination with standard thyrostatic drugs would be a logical treatment extension (3).

\section{Methods}

Based on a pre-specified protocol, we searched PubMed, EMBASE and Cochrane (all up to Jul 2015). We searched for (randomized)-controlled trials comparing immunosuppressive drugs with a control group. PRISMA and SIGN statements were used for reviewing data and assessing quality. Two reviewers extracted data on study characteristics, methods, and outcomes. Data were pooled using a random-effects model. The primary endpoint was relapse of disease until follow-up, secondary endpoints included reduction of thyroid volume and decrease in TSH-receptorantibody [TRAb] levels.

\section{Results}

\begin{tabular}{|c|c|c|c|c|c|}
\hline \multicolumn{6}{|c|}{ Primary endpoint } \\
\hline Overall analysis & $113 / 481(23.5 \%)$ & $225 / 381(59.1 \%)$ & $0.55(0.41,0.75),(p<0.001)$ & $74 \%$ & \\
\hline \multicolumn{6}{|c|}{ Subgroups - Type of immunosuppressive drug used } \\
\hline Other immunosuppressives & 9/46 (19.6\%) & 25/38 (65.8\%) & $0.42(0.15,1.17)$ & $20 \%$ & $0 \%$ \\
\hline \multicolumn{6}{|c|}{ Subgroups- Type of study } \\
\hline \multicolumn{6}{|c|}{ Secondary endpoints - mean difference $(95 \% \mathrm{Cl})$} \\
\hline Thyroid volume & \multirow{2}{*}{$292(60.1 \%)$} & \multirow{2}{*}{$194(39.9 \%)$} & $-10.72 \mathrm{ml}(-15.59,-5.85)$ & $100 \%$ & \\
\hline TRAb levels & & & $-17.01 \mathrm{U} / \mathrm{L}(-33.31,-0.72)$ & $100 \%$ & \\
\hline
\end{tabular}

\section{Forest Plot for Relapse Risk}

We included 7 trials with 862 participants. Most trials were small with moderate to high risk of bias. There were 113 relapses in 481 (23.5\%) patients receiving immunosuppressive drugs compared to 225 relapses in 381 (59.1\%) control patients (risk ratio for recurrence $0.55,95 \%$ confidence interval $[\mathrm{Cl}] 0.41,0.75$ ). Subgroup analyses showed similar effects for randomized trials and controlled trials (I2 $0 \%$ ), as well as for trials using corticosteroids (hydrocortisone, dexamethasone, methyl-prednisolone, prednisolone) and noncorticosteroids (azathioprine, rituximab, cyclophosphamide) immunosuppressive drugs (12 0\%). Immunosuppressive drug-related adverse effects were not systematically reported and thus not included in the quantitative analysis. They included leucopenia, rash, minor infections, chills and fever during infusion of rituximab. There were no reports on serious adverse effects.

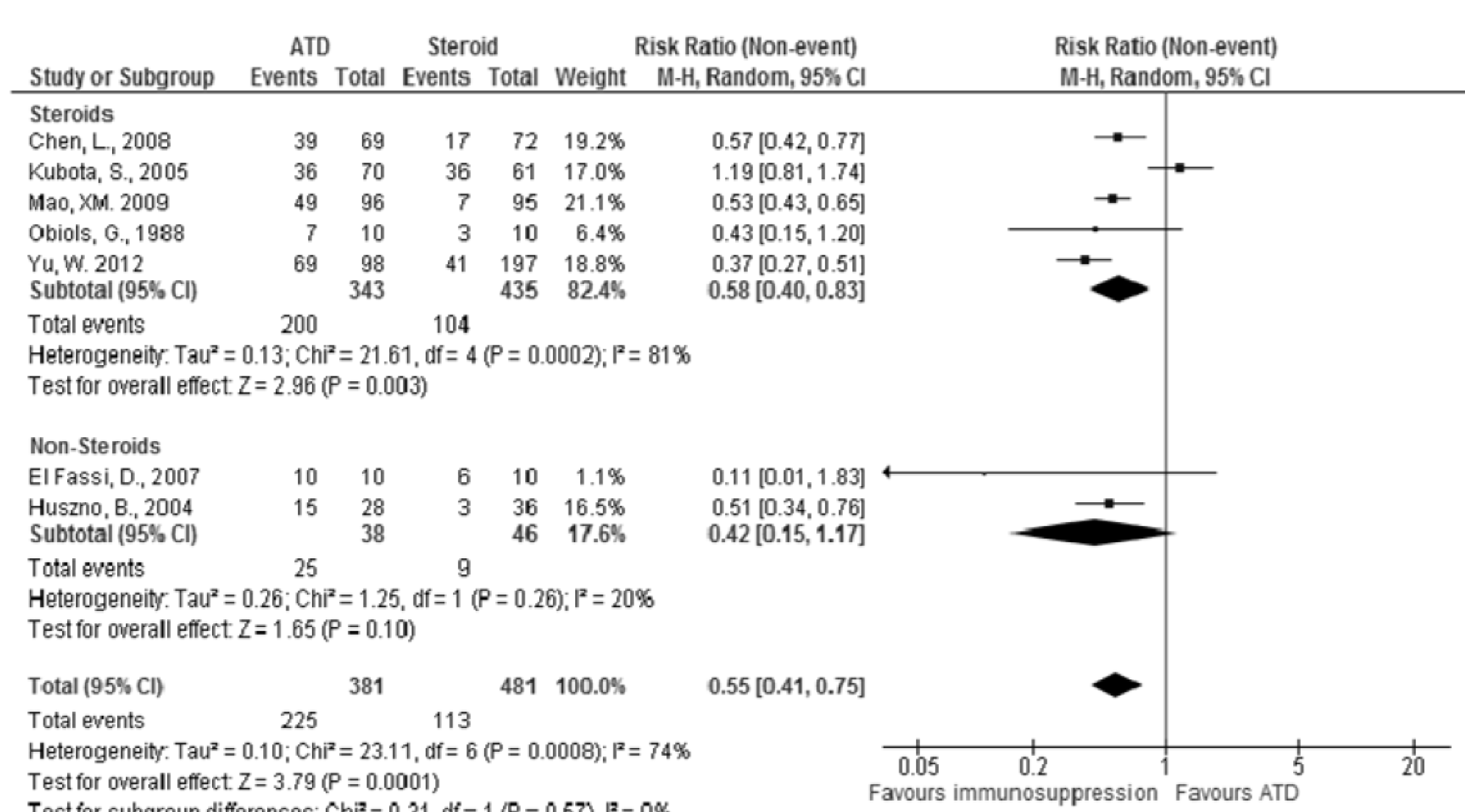

\section{Forest Plots for Reduction of Thyroid Volume (upper) and TRAb (lower)}

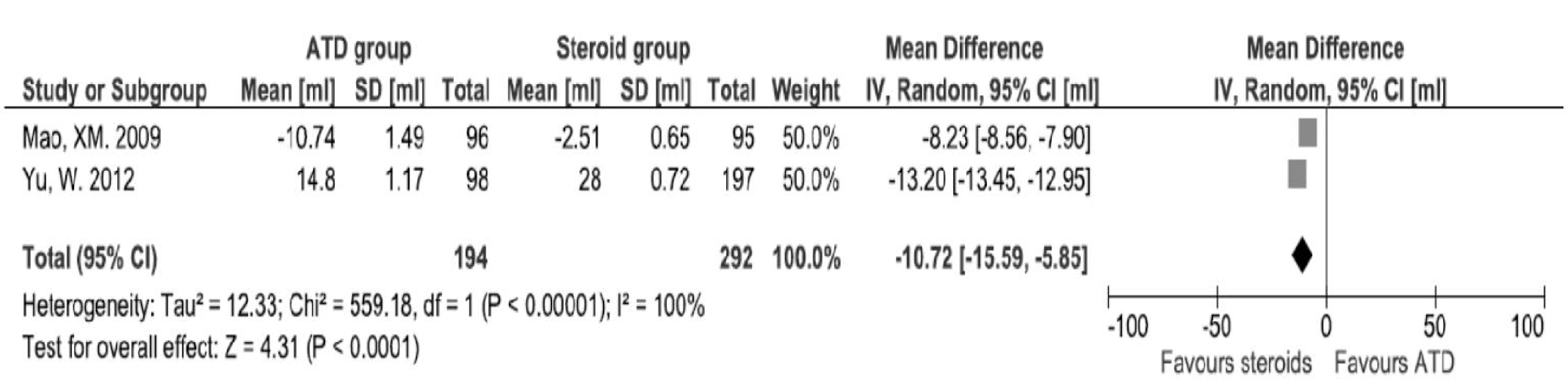

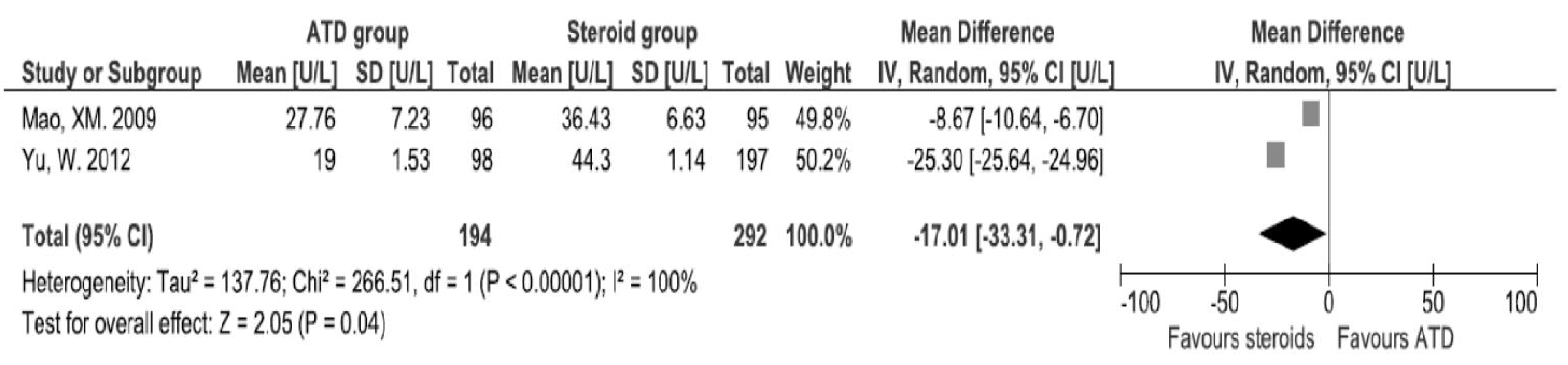

\section{Conclusion}

Current evidence suggests a relevant reduction in relapse risk when immunosuppressive drugs are added to standard treatment of Graves' disease. The small number of trials with high heterogeneity and the lack of systematic reporting of adverse effects calls for larger, conclusive trials.

\section{References}

(1) Genovese BM, et al., 2013 What is the best definitive treatment for Graves' disease? A systematic review of the existing literature. Ann. Surg. Oncol. 20: 660-667. (2) Antonelli A, Ferrari SM, Corrado A, Di Domenicantonio A, Fallahi P 2015 Autoimmune thyroid disorders. Autoimmun Rev 14: 174-180.

(3) Franklyn JA, Boelaert K 2012 Thyrotoxicosis. Lancet 379: 1155-1166.

Email: tristan.struja@gmail.com 